\title{
Biomechanical effects of different vertebral heights after augmentation of osteoporotic vertebral compression fracture: a three- dimensional finite element analysis
}

\author{
Wen-Tao Zhao ${ }^{1,3+}$, Da-Ping Qin ${ }^{1,2+}$, Xiao-Gang Zhang ${ }^{1,2^{*}}$, Zhi-Peng Wang ${ }^{1,2}$ and Zun Tong ${ }^{1,2}$
}

\begin{abstract}
Background: Clinical results have shown that different vertebral heights have been restored post-augmentation of osteoporotic vertebral compression fractures (OVCFs) and the treatment results are consistent. However, no significant results regarding biomechanical effects post-augmentation have been found with different types of vertebral deformity or vertebral heights by biomechanical analysis. Therefore, the present study aimed to investigate the biomechanical effects between different vertebral heights of OVCFs before and after augmentation using three-dimensional finite element analysis.
\end{abstract}

Methods: Four patients with OVCFs of T12 underwent computed tomography (CT) of the T11-L1 levels. The CT images were reconstructed as simulated three-dimensional finite-element models of the T11-L1 levels (before and after the T12 vertebra was augmented with cement). Four different kinds of vertebral height models included Genant semiquantitative grades $0,1,2$, and 3, which simulated unilateral augmentation. These models were assumed to represent vertical compression and flexion, left flexion, and right flexion loads, and the von Mises stresses of the T12 vertebral body were assessed under different vertebral heights before and after bone cement augmentation.

Results: Data showed that the von Mises stresses significantly increased under four loads of OVCFs of the T12 vertebral body before the operation from grade 0 to grade 3 vertebral heights. The maximum stress of grade 3 vertebral height pre-augmentation was produced at approximately 200\%, and at more than 200\% for grade 0 . The von Mises stresses were significantly different between different vertebral heights preoperatively. The von Mises stresses of the T12 vertebral body significantly decreased in four different loads and at different vertebral body heights (grades 0-3) after augmentation. There was no significant difference between the von Mises stresses of grade 0,1 , and 3 vertebral heights postoperatively. The von Mises stress significantly decreased between pre-augmentation and post-augmentation in T12 OVCF models of grade 0-3 vertebral heights.

Conclusion: Vertebral augmentation can sufficiently reduce von Mises stresses at different heights of OVCFs of the vertebral body, although this technique does not completely restore vertebral height to the anatomical criteria.

Keywords: Vertebral augmentation, Von Mises stress, Osteoporotic vertebral compression fracture

\footnotetext{
*Correspondence: zxg0525@163.com

${ }^{\dagger}$ Equal contributors

'Gansu University of Chinese Medicine, No. 35, Dingxi East Rd., Chengguan

District, Lanzhou 730000, Gansu Province, People's Republic of China

${ }^{2}$ Affiliated Hospital of Gansu University of Chinese Medicine, No. 735,

Jiayuguan West Rd., Chengguan District, Lanzhou 730000, Gansu Province,

People's Republic of China

Full list of author information is available at the end of the article
} 


\section{Background}

Osteoporotic vertebral compression fracture (OVCF) is a common disease in the elderly population accompanied by decreased bone mineral density [1], which can cause acute or chronic back pain, functional limitations of the spine, a thoracolumbar vertebral deformity, vertebral height $(\mathrm{VH})$ loss, and deterioration of quality of life $[2,3]$. OVCFs occur more frequently than other osteoporotic fractures, such as hip fractures and distal radius fractures [4], and they have become a more increasingly serious disease and a significant health problem worldwide that will obviously increase economic burden to society and family in the future [5].

Before vertebral augmentation (VA) techniques were developed, conservative treatment options of OVCFs included bed rest, analgesic drugs, calcium supplementation, antiresorptive drugs, and a spine brace for a few weeks. To stabilize fractures quickly, relieve pain fast, and achieve restoration of $\mathrm{VH}$ for the treatment of OVCFs, two minimally invasive VA procedures, percutaneous vertebroplasty (PVP) and percutaneous kyphoplasty (PKP), have been performed by percutaneously injecting bone cement into the fractured vertebral body $[6,7]$. The difference between these procedures is that PVP is used to stabilize the fracture primarily through a very small skin incision in which bone cement is injected, and PKP can achieve more restoration of $\mathrm{VH}$ through insertion of a balloon through the same skin incision and expansion of the fractured vertebral body before the cement injection [8]. Two kinds of bone cement injection methods are used in PKP and PVP: unilateral or bilateral injection. The benefit of these minimally invasive procedures compared to conservative treatment or open surgery is better pain relief and functional spine improvement [4]. When bone cement is injected into the vertebral body, it may have analgesic effects by consolidating micro-fractures and reducing the mechanical stress generated by body weight and with activity, and it may destroy bone nerve endings by a cytotoxic and exothermal action in the course of cement polymerization [9].

Biomechanical tests of PKP have confirmed that cement augmentation improved vertebral fracture stability, and there were no significant differences in $\mathrm{VH}$ restoration in in vitro studies $[10,11]$. The results of restoration of mechanical stability through VA have been also confirmed by finite element analysis (FEA), which can predict the long-term stability of a bone after cement augmentation through reliable models [8, 12].

Many previous studies have reported excellent clinical results of augmentation with PVP and PKP, which strengthen augmented vertebral bodies and can result in significant, rapid pain relief in $80-90 \%$ of patients, stabilize vertebral compression fractures quickly, and improve spinal deformity $[1-3,5]$. According to clinical measurements on vertebral compression fractures treated with PVP or PKP, a certain amount of height restoration is achieved only in $66 \%$ of treated patients [8]. $\mathrm{VH}$ and kyphosis deformities can result in different recovery and correction, but pain and function can be adequately improved. Many surgeons think that the treatment of fractures requires good reduction, as the shift in the fracture segment may affect healing and function; however, several prior analyses have focused on total pain relief, quality of life, and safety outcomes and did not observe the effects between the different heights of the vertebral body after VA [13-15]. Therefore, it is necessary to explore the effect of vertebral strength on different VHs. Although there are many ways to classify OVCFs, the distinction between changes of $\mathrm{VH}$ is a more intuitive way to classify these fractures. Clinical results have shown that different VHs have been restored postoperatively and the treatment results are consistent. Currently, no significant results regarding biomechanical effects post-augmentation have been reported with different VHs by biomechanical analysis. FEA is likely to be the gold standard as an alternative to bone strength research methods [16], and it may help assess vertebral fracture stability as a biomechanical analysis [17]. The aim of the present study was to explore the biomechanical effects between different VHs of OVCFs before and after augmentation by three-dimensional (3D) FEA. By analyzing the differences in stress changes, we will determine the consistency of biomechanical results and clinical findings for deciding whether good reduction must be performed through surgery.

\section{Methods}

This study was granted an exemption from requiring ethics approval by the ethics committee of the Affiliated Hospital of Gansu University of Chinese Medicine. The authors obtained patient consent before enrolling participants in this study.

\section{Patients of the FEA models of T12 OVCFs}

In this study, four female volunteers with T12 OVCFs underwent computed tomography (CT) from the T11 level to the L1 level after VA, with a slice thickness of $0.625 \mathrm{~mm}$. The CT images were reconstructed to simulate 3D FEA models. Inclusion criteria of OVCFs were a fracture due to acute minor or mild external trauma, individuals with a visual analog scale (VAS) score for back pain $\geq 5$, the affected vertebral body showed a hypointense signal on T1-weighted magnetic resonance imaging scans and hyperintense signal on T2-weighted magnetic resonance imaging scans, and those with a $T$ score $\leq-1.5$ for bone mineral density detected by dualenergy X-ray absorptiometry. The exclusion criterion was individuals with other pathological fractures 
detected by radiography. VHs of postoperative CT images were evaluated by Genant semi-quantitative grades $0-3$. Vertebral bodies were graded as normal (grade 0 ), mildly deformed (grade 1 , approximately $20-25 \%$ reduction anteriorly), moderately deformed (grade 2, approximately $25-40 \%$ reduction in any height and a reduction in area of $20-40 \%$ ), and severely deformed (grade 3, approximately $40 \%$ reduction in any height and area) [18]. Crosssection images of the four different grades are shown in Fig. 1a-h. All CT images were saved in Digital Imaging and Communications in Medicine (DICOM) format.

\section{The construction of T11-L1 3D models and T12 fracture models}

Four 3D models were developed based on the CT scans of T11-L1 vertebral bodies. The CT images of 4 patients in DICOM format were imported into Materialise Interactive Medical Image Control System (Mimics, version 10.01; Materialise, Inc., Leuven, Belgium) to generate the 3D model of the T11-T12-L1 vertebral bodies, including the cortical (1 mm thick) and cancellous bone. The following steps from the segmentation menu were performed: threshold segmentation was used to separate the bone and soft tissue, and editing mask tools were used to edit the image shape, select the desired area, fill the image area appearing in the gap, and split out the required contour layer by layer. Finally, 3D models were reconstructed through the edit mask option. The reconstructed 3D models were saved in STL format.

STL models were imported into automatic reverse engineering software, Geomagic studio (version 2012; Geomagic, Raindrop Geomagic, Research Triangle Park, NC, USA) for smooth noise reduction, feature removal, and structural patches and fitting surfaces. Then, the surface of the 3D models of the vertebral bodies were generated and saved in STP format. The models were optimized using Geomagic studio software and imported into Solidworks (version 2012; Dassault Systems, SolidWorks Corp., Santa Monica, CA), where T11, T12, L1 vertebral bodies were assembled for transposition and the surface command was used to generate the end-plate, cartilage, and intervertebral disc (nucleus pulposus and annulus). The desired 3D model was obtained by assembling these structures. Using a previously reported simulation method, models of the T12 fracture line were produced using the surface command to cut the vertebral body to produce the $0.5-\mathrm{mm}$ fracture line (Fig. 2) [19].

\section{The construction of T12 models post-augmentation}

STP models were imported into Solidworks software. Unilateral bone cement injection models were produced by software; a 4-mL upright pillar similar to a bone cement model was used; the bone cement in the T12 vertebral model was assembled in the center using the assembly command; and then through the software's Boolean operation function, excess bones were removed, and the bone cement model was assembled into the vertebral body (Fig. 3). The 3D model of the T12 vertebral body after bone cement augmentation was obtained. Finally, the various models that included the T12-L1 vertebral bodies, bone cement, intervertebral discs, end-plates, and cartilages were assembled by the software to generate integral $3 \mathrm{D}$ parts.

\section{FEA models}

The material properties used in recent studies about OVCFs are shown in Table 1 [20-23]. FEA was performed using ANSYS software (version 17.0, ANSYS, Canonsburg, PA, USA). The 3D models, including the cortical and cancellous bone cement, end-plate, cartilage, and intervertebral disc (nucleus pulposus and annulus), were imported into ANSYS software.
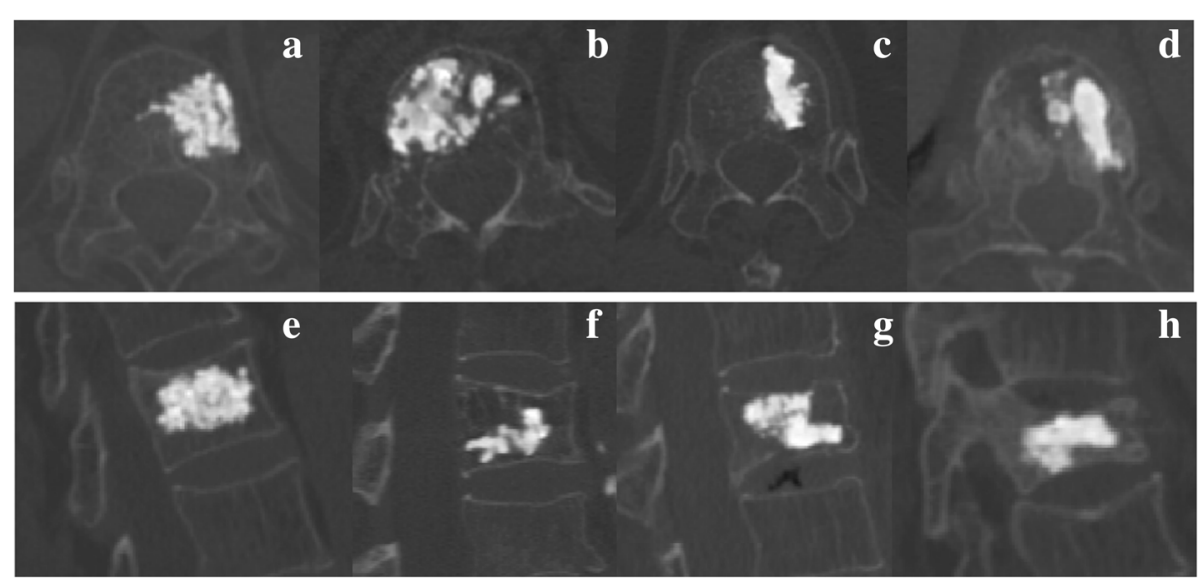

Fig. 1 Computed tomography images of 4 patients with T12 osteoporotic vertebral compression fractures after vertebral augmentation. a-d Cross-section images of grade 0-3 vertebral height; $\mathbf{e}-\mathbf{h}$ sagittal images of grade $0-3$ vertebral height 


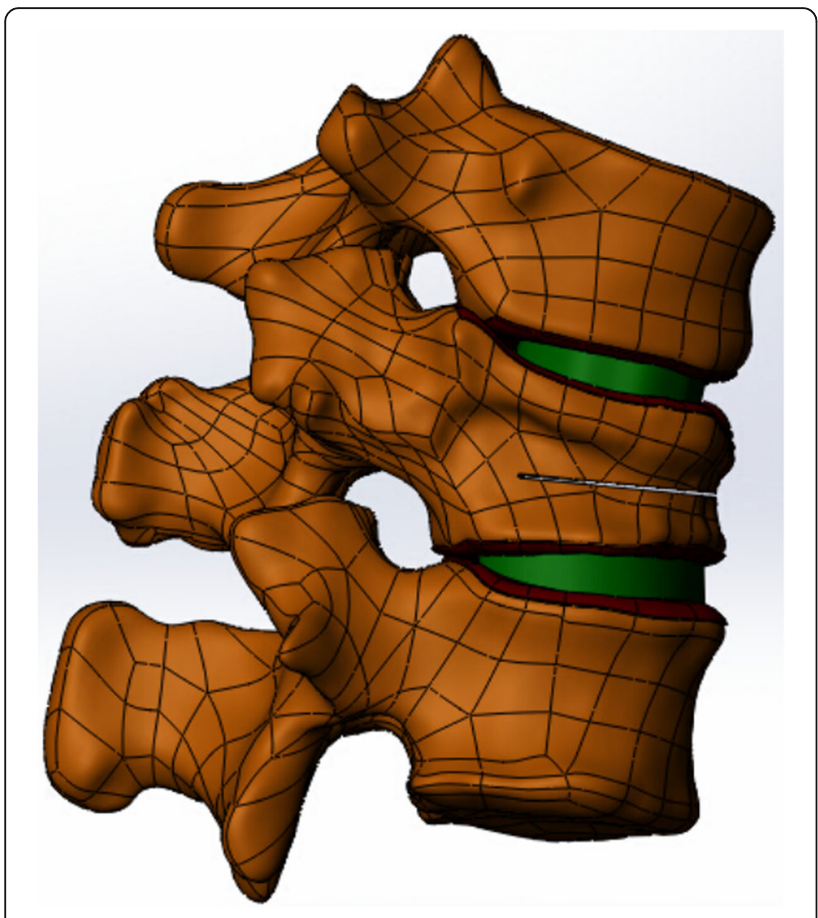

Fig. 2 T11-L1 three-dimensional model and T12 fracture model

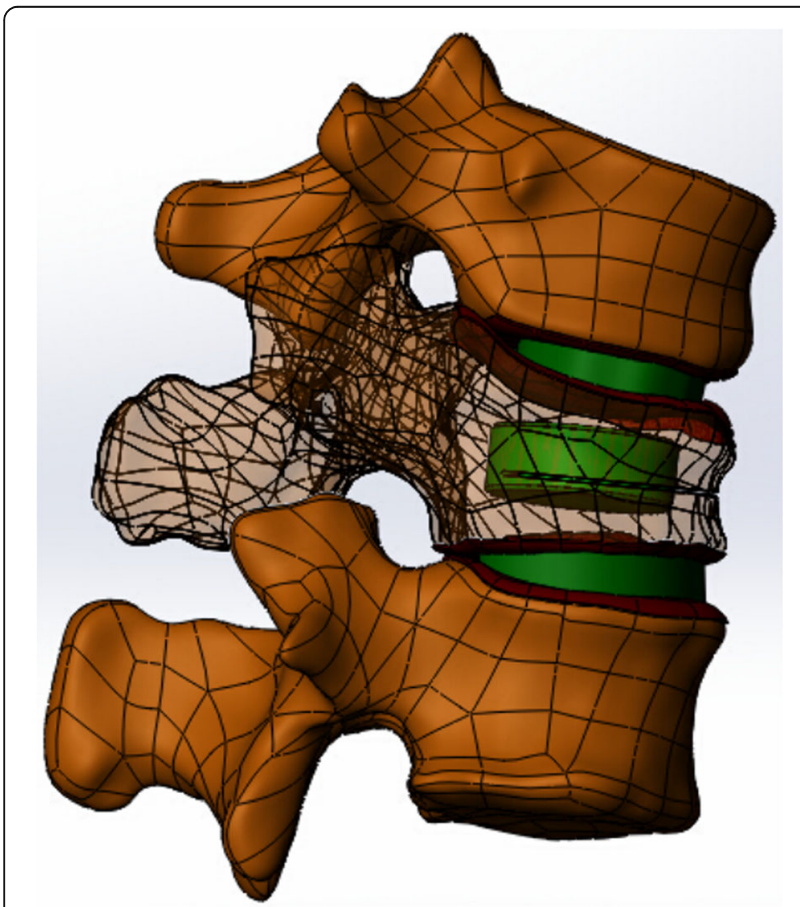

Fig. 3 T11-L1 three-dimensional model and the T12 vertebral body after augmentation
Table 1 Material properties of finite element analysis models

\begin{tabular}{lll}
\hline Component & Young modulus (MPa) & Poisson ratio \\
\hline Cortical bone & $8040(67 \%$ normal $)$ & 0.3 \\
Cancellous bone & $34(34 \%$ normal $)$ & 0.25 \\
Bony end-plate & $670(67 \%$ normal $)$ & 0.4 \\
Annulus & 4.2 & 0.45 \\
Nucleus pulposus & 1 & 0.4999 \\
Cartilage & 10 & 0.4 \\
Cement & 3000 & 0.4 \\
ALL & 20 & 0.3 \\
PLL & 70 & 0.3 \\
ISL & 28 & 0.3 \\
SSL & 28 & 0.3 \\
LF & 50 & 0.3 \\
CL & 26 & 0.3
\end{tabular}

ALL anterior longitudinal ligament, $P L L$ posterior longitudinal ligament, ISL interspinous ligament, SSL supraspinal ligament, $L F$ ligamentum flavum, $C L$ capsular ligament

Supplemental components included the anterior longitudinal ligament (ALL), posterior longitudinal ligament (PLL), interspinous ligament (ISL), supraspinal ligament (SSL), ligamentum flavum (LF), and capsular ligament (CL). All models and bone cement were assigned linear elastic isotropic material properties. The element types of cortical bone, cancellous bone, bony end-plate, facet joint cartilage, and nucleus pulposus were defined as solid elements with a material representation of linear isotropic elasticity. The element types of the ALL, PLL, ISL, SSL, CL, and LF enable tension deformation without compression behavior. The end-plate, cartilage, and intervertebral disc (nucleus pulposus and annulus) were divided into 2-mm mesh. Cortical and cancellous bone cement were divided into 5 - $\mathrm{mm}$ mesh. The mesh, nodes, and units are self-generated by the software. Connections between the end-plate and vertebral body, end-plate and intervertebral disc, and cartilage and bones were bonded. Connections between the cartilage and cartilage were frictionless. The lower edge of the L1 vertebral was set to fixed. The loads of vertical compression, flexion, and right lateral bending, or left lateral bending with four different loads were separately applied from the T11 vertebral upper edge. The pre-process for FEA was performed using meshed models (i.e., a meshing element was used, and material properties and boundary conditions were applied) (Fig. 4).

\section{FEA}

Static FEA was performed by simulating the different extent of the fracture using four grades of $\mathrm{VH}$ of the T12 OVCF and two kinds of models: fresh OVCF (preaugmentation) and OVCF after cement augmentation 


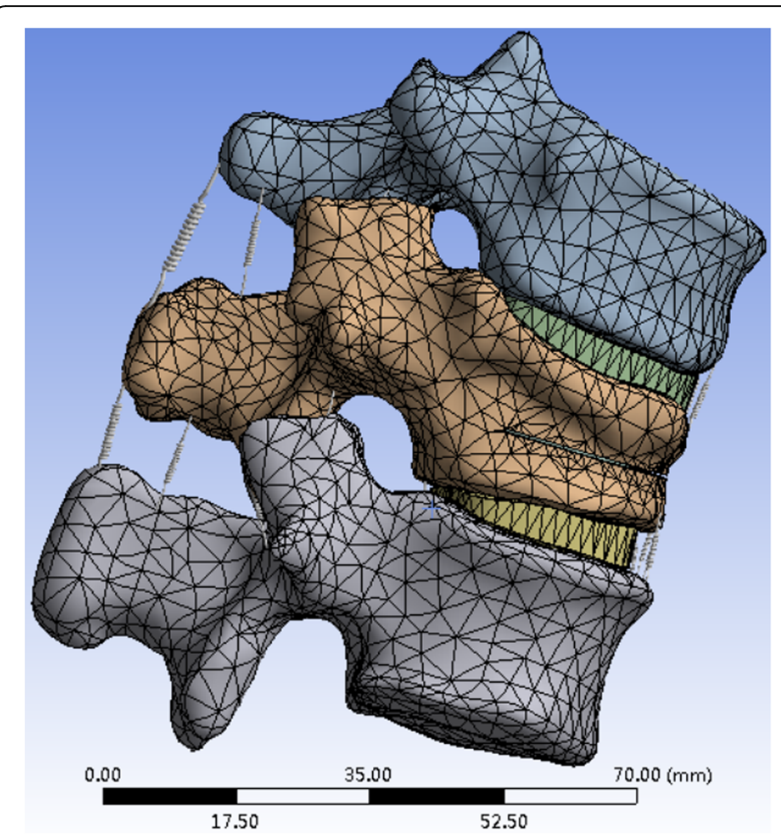

Fig. 4 T11-L1 finite element analysis model

(post-augmentation). Five hundred newtons for vertical compression load and 7.5 N.m moment were applied for vertical compression and flexion, left flexion, and right flexion in all models. According to the spinal threecolumn concept, the load and moment were applied to the superior end-plate and articular facets of the T11 vertical body, with $85 \%$ of these on the anterior-middle column and $15 \%$ on the posterior column [19, 24-26]. Since the aim of this study was to evaluate the overall biomechanical changes of the vertebral body, the overall von Mises stresses on the T12 vertical bodies were calculated to evaluate the effects of cement augmentation. These FEA models of the T11-L1 vertebral bodies under vertical flexion and right or left flexion load were verified similarly to those published in the literature [27-29]. The normal model was validated according to published FEA models of human cadaveric thoracolumbar spines by ANSYS [23, 29]. Results of stress on the T12 vertebral body and stress cloud images at the end of the analysis can be exported to a computer. However, in this study, images of the T12 vertebral body and upper end-plate under stress are shown because the loads were conducted from the top to the bottom.

\section{Statistical analysis}

The von Mises stresses on T12 vertebral bodies pre-augmentation and post-augmentation were applied under vertical compression and flexion, left flexion, and right flexion loads. One-way analysis of variance was used to analyze the effect of different loads and VHs before and after augmentation. SPSS software (version 17.0, IBM
Corp., Armonk, NY, USA) was used to perform all statistical analyses. $P<0.05$ was considered statistically significant.

\section{Results}

Participants' ages were $63,71,77$, and 84 years, and VAS scores were $6,8,7$, and 6 , respectively. All patients underwent unilateral PKP. VAS scores decreased to 2, 2, 2 , and 1 on the second day after surgery.

\section{Stress on the T12 vertebral body for different VHs pre-augmentation}

Results were analyzed by four models of T12 OVCFs with grade $0-3 \mathrm{VHs}$ before augmentation. The von Mises stresses of different VHs of T12 OVCFs under vertical compression and flexion, left flexion, and right flexion are shown in Figs. 5 and 6. Under vertical compression load, von Mises stresses on the T12 vertebral body for grades $0,1,2$, and 3 were 33.282, 49.84, 59.93, and $68.966 \mathrm{MPa}$, respectively. Similarly, the von Mises stresses increased under flexion load from 101.89 $\mathrm{MPa}$ to $181.93 \mathrm{MPa}$. Regarding the different VHs, stresses of fresh OVCFs of the T12 vertebral body had a similar trend of change, which was that more height loss increased stress. The maximum stress of grade $3 \mathrm{VH}$ preaugmentation was produced at approximately $200 \%$ in flexion and left flexion, and at more than $200 \%$ for grade 0 in vertical compression and right flexion. There were significant differences in the loads between different VHs preoperatively.

\section{Stress on the T12 vertebral body for different VHs post-augmentation}

The von Mises stresses of different VHs postaugmentation of the T12 vertebral body under vertical compression and flexion, left flexion, and right flexion are shown in Figs. 7 and 8. Augmentation significantly decreased the stress on the T12 vertebral body in all four different loads for grade $0-3 \mathrm{VH}$. The maximum stress under flexion was $10.505 \mathrm{MPa}$ for grade $3 \mathrm{VH}$. The maximum stress under all loads was $10.575 \mathrm{MPa}$ in flexion for grade $1 \mathrm{VH}$. A comparison of postoperative loads among the four grades of $\mathrm{VH}$ showed that there was a significant difference between grades 0,1 , and 3 and grade 2 , and no significant difference between grade 0 and grades 1 and 3 . The trend seen pre-augmentation (i.e., more height loss and increased stress) changed.

\section{The von Mises stresses on fresh OVCFs before and after augmentation}

A comparison of the von Mises stress in T12 OVCF models of grade $0-3 \mathrm{VHs}$ before and after augmentation is shown in Fig. 9 and Table 2. All von Mises stresses were significantly decreased post-augmentation for 


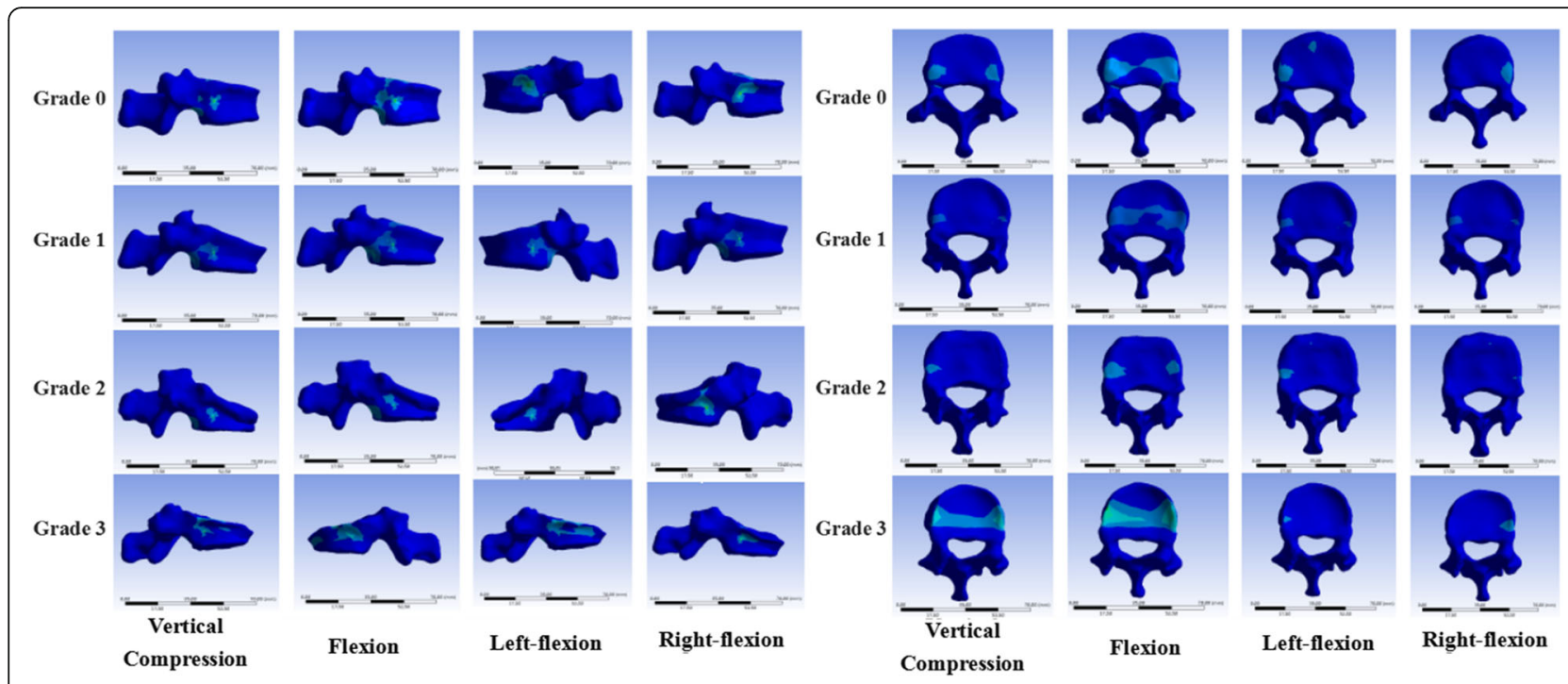

Fig. 5 Nephograms of the von Mises stresses on fresh T12 osteoporotic vertebral compression fractures. The side and upper end-plate are shown

different VHs. There were significant differences in preoperative and postoperative loads. The most obvious reduction and most significant change was observed for grade $3 \mathrm{VH}$, from $181.93 \mathrm{MPa}$ to $7.9141 \mathrm{MPa}$ under flexion load. The trend of changes of stress in various conditions was not so obvious postoperatively as it was preoperatively.

\section{Discussion}

OVCF is an important health issue in the aging population, and it is a common fragility fracture involving the collapse, compression, or wedging of a vertebral body, which may be accompanied by a lasting, painful, and disabling condition of the spine [30]. Owing to the increase in prevalence of osteoporosis and life expectancy of humans, the incidences of OVCFs are also increasing [31]. Although conservative therapy of OVCFs is performed routinely, it is difficult for patients to tolerate long-term bed rest. The traditional surgical treatment is accompanied by a similar disadvantage, which may cause various complications, such as pneumonia, urinary tract infection, bedsores, and deep venous thrombosis, especially among elderly patients [32]. Treatment of OVCFs needs to restore the $\mathrm{VH}$. However, due to the effects of osteoporosis and stress, some patients do not experience restored $\mathrm{VH}$ at all after clinical treatment. Although PKP and PVP can relieve the clinical symptoms of patients with OVCFs through VA, the VH of these patients cannot be fully reduced [6, 7], and there are no acceptable clinical reduction criteria. The present study showed that the vertebral bodies can be bear similar stress after VA, as they did not have more height loss or bear more stress. This result is consistent with that of other clinical studies.

The severity of fractures and vertebral deformities can be classified by images of the spine using several methods. The semi-quantitative technique of Genant is one of the most common methods. However, this

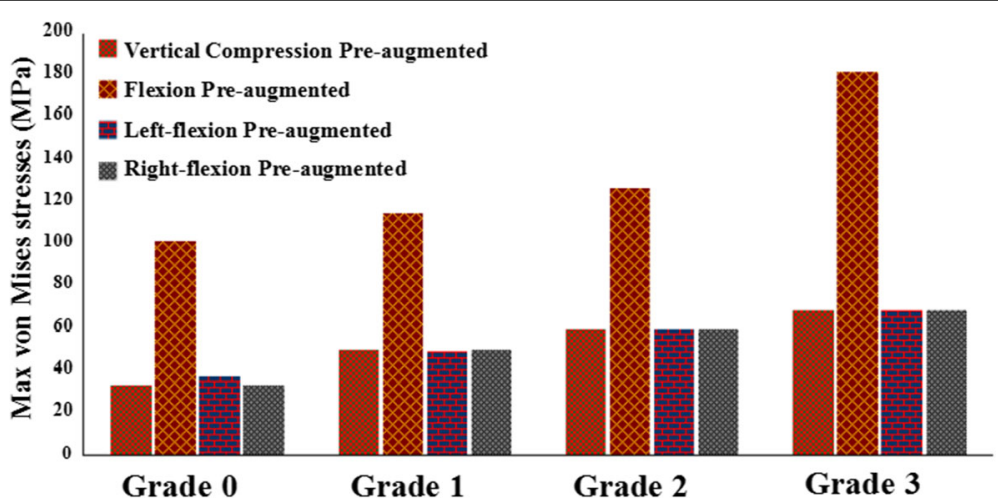

Fig. 6 The von Mises stresses on fresh T12 osteoporotic vertebral compression fractures 


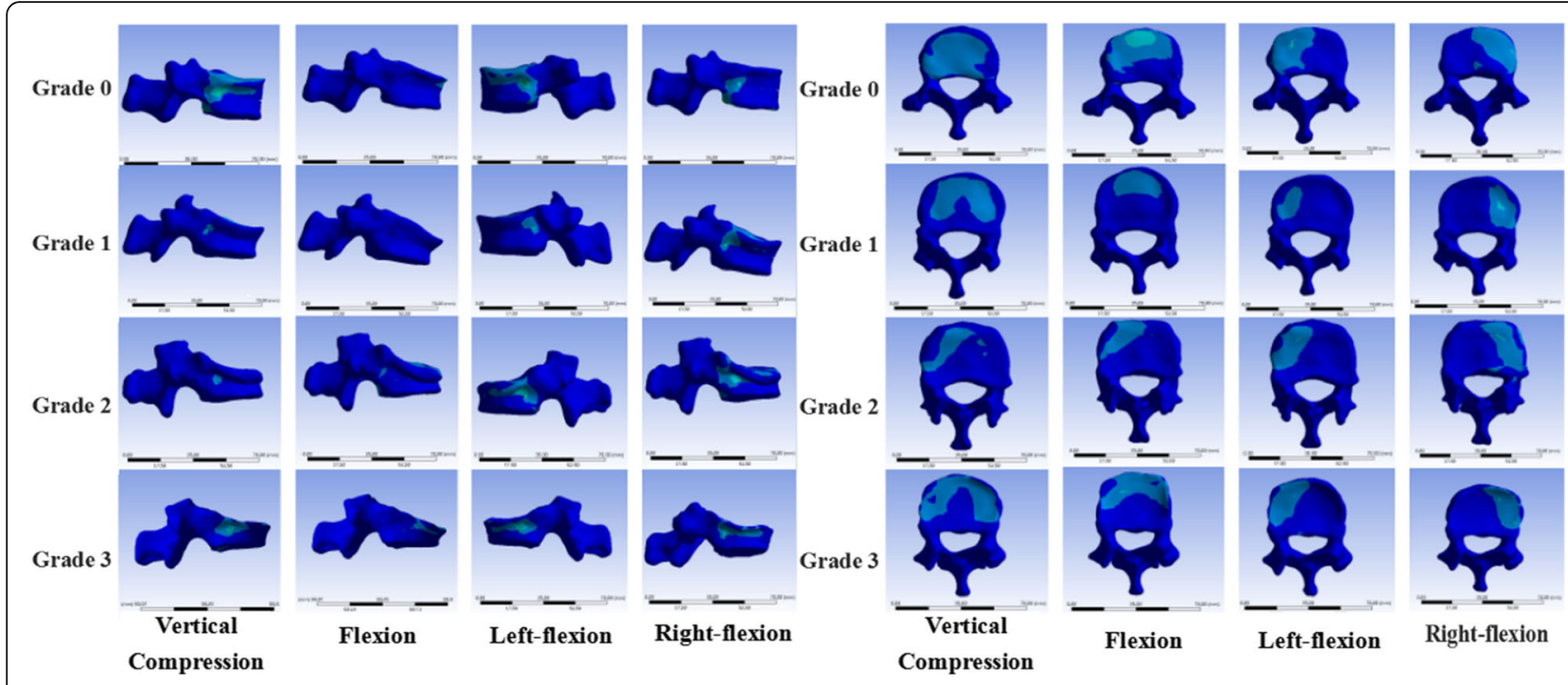

Fig. 7 Nephograms of the von Mises stresses on T12 osteoporotic vertebral compression fractures after augmentation. The side and upper end-plate are shown

preoperative classification has only a small predictive power for postoperative reductions [3]. Due to the aging of patients and a low bone density, there are several difficulties in treating OVCFs through surgical methods, which may result in loose internal fixation, fracture recurrence, etc. However, VA techniques, including PVP and PKP, are efficacious surgical treatment methods for OVCFs [33], and they have been widely used to treat OVCFs to relieve back pain and correct deformity of the spine. PVP and PKP have resulted in significant pain relief and an increase in life expectancy, as well as improvement in spine function [34]. Studies have not found any significant differences between PVP and PKP in pain relief, mental health, and movement of the spine. Pain relief was the main outcomes in all research studies of PVP and PKP. As one of the criterion for evaluating the effect of fracture treatment, restoration of the bone position should be assessed using preoperative and postoperative images. PVP and PKP can achieve a significant increase in $\mathrm{VH}$ and a significant reduction in the kyphotic wedge angle [35]. For the treatment of OVCFs, VA techniques serve as minimally invasive and effective surgical methods, although the extent of $\mathrm{VH}$ restoration is not consistent postoperatively.

It has also been shown that VA cannot completely restore the VH by PVP and PKP based on a comparison of radiographs and $\mathrm{CT}$ images. In our research study, the clinical results of preoperative and postoperative images of semi-quantitative grading of $\mathrm{VH}$ were not significantly different, but pain relief was confirmed, although the operation could not completely restore the VH. Experimental biomechanical studies have shown that PVP or PKP increased stiffness and strength [36, 37]. Moreover, the increased stiffness of the augmented vertebral body led to an increase in the load of adjacent vertebral bodies [38], increased failure load of the vertebral body after augmentation [39], improved the degree of $\mathrm{VH}$ restoration after augmentation, and increased the risk of

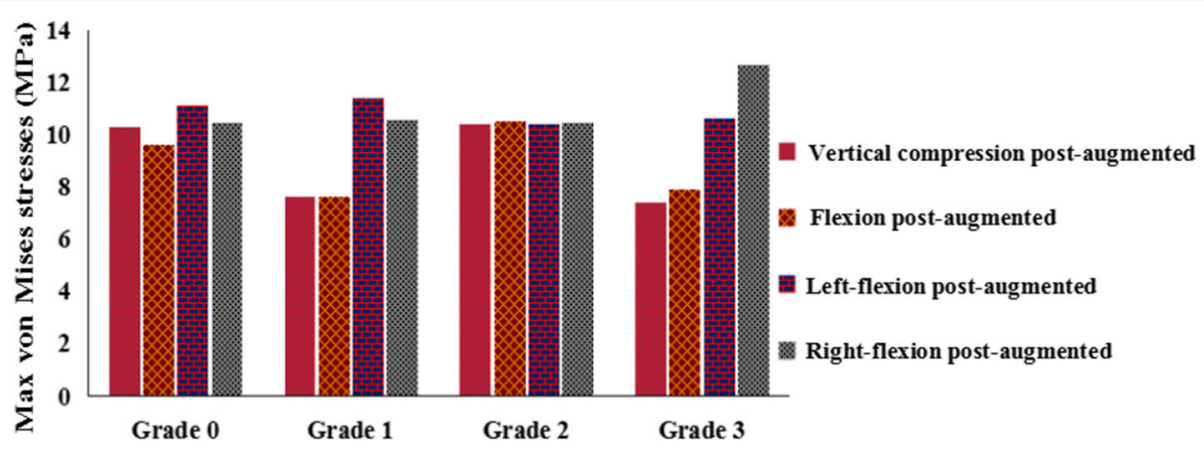

Fig. 8 The von Mises stresses on the T12 vertebral body after augmentation 


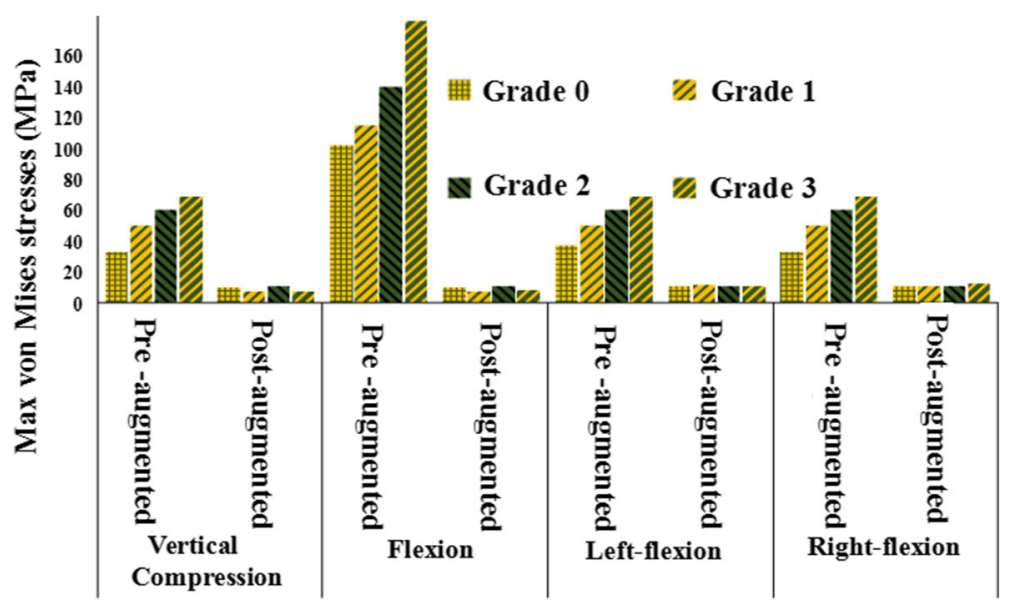

Fig. 9 Comparison of the von Mises stresses before and after augmentation

new fracture [40]. Additionally, the volume and distribution of biological materials, such as bone cement, used in surgery may have a significant effect in studies of the mechanical load of the models [41, 42]. The result of FEA showed that augmentation of bone cement can increase the risk of adjacent vertebral fractures [36]. FEA also demonstrated that an increased volume of bone cement can have a significant effect on the occurrence of subsequent vertebral fractures after augmentation [43]. The Von Mises law is a theory used to analyze the stress distribution on materials and models. According to this theory, the stress produced is called the von Mises stress and it has been recently used for evaluating the stress distribution on important areas of the virtual model [44]. These results of von Mises stress suggest that there are some important biomechanical phenomena outside the results of good clinical studies. However, these studies did not specifically study the biomechanical changes of different VHs after VA. In our study, although the preoperative and postoperative reductions of $\mathrm{VH}$ were different in grade $0-3 \mathrm{VH}$, VA significantly reduced the stress on the vertebral body under different loads and VHs.

In the current study, four different VHs in FEA models of OVCFs were established based on measurements from postoperative CT images. Since unilateral and bilateral percutaneous augmentation can provide excellent pain relief and improvement of life quality [4], unilateral bone cement injection models were used. Results of the FEA method provided the following biomechanical evidence: with clinical treatment, the VH cannot be fully restored after surgical treatment. In the four models, all biomechanical parameters, including the Poisson ratio and elastic modulus, were used according to previous literature [20-23]. Compared with other FEAs, we used four FEA models of different VHs. Results of FEA showed that the greater the loss of $\mathrm{VH}$, the greater the stress on the vertebral body before vertebral reinforcement; additionally, vertebral stress of different VHs was significantly reduced.

The limitations of these FEA models must be considered. In this study, because the shapes of cement in the postoperative vertebral body were not exactly the same, we did try to use the real cement augmentation model for FEA. However, we found that we could not control the different effects of different cement forms on the experimental results. The use of a cylindrical cement model is easy for computer calculations and ensures the repeatability of the study [19]. This study's findings do not reflect the complexities of real-life situations since several factors were not considered (e.g., the FEA models were too simplified; multiple vertebral fractures were

Table 2 The von Mises stresses (MPa) on the T12 vertebral body

\begin{tabular}{|c|c|c|c|c|c|c|c|c|}
\hline & \multicolumn{2}{|c|}{ Vertical compression } & \multicolumn{2}{|l|}{ Flexion } & \multicolumn{2}{|c|}{ Left flexion } & \multicolumn{2}{|c|}{ Right flexion } \\
\hline & Pre & Post & Pre & Post & Pre & Post & Pre & Post \\
\hline Grade 0 & 33.282 & 10.252 & 101.89 & 9.6296 & 37.54 & 11.08 & 33.159 & 10.433 \\
\hline Grade 1 & 49.84 & 7.6211 & 115.07 & 7.641 & 49.831 & 11.39 & 49.893 & 10.575 \\
\hline Grade 2 & 59.93 & 10.392 & 139.52 & 10.505 & 59.931 & 10.396 & 59.927 & 10.431 \\
\hline Grade 3 & 68.966 & 7.3754 & 181.93 & 7.9141 & 68.97 & 10.629 & 68.954 & 12.681 \\
\hline
\end{tabular}

Pre pre-augmented, post post-augmented 
excluded; and muscle force and the bone cement shape and location postoperatively were not considered). All these factors could have affected the von Mises stress observed before and after augmentation. Since the anatomical morphology of the four models was inconsistent, the stress of different vertebral bodies may also lead to subtle changes in stress trends despite the use of the same loading method. The result of the comparison of postoperative loads between the four grades of $\mathrm{VHs}$ may have been affected by this limitation; there was a significant difference between grades 0,1 , and 3 and grade 2, rather than no significant difference between all four grades. Therefore, the biomechanical changes in the clinical setting cannot be fully understood by virtue of this analysis. Based on the present study, a future biomechanical analysis should include FEA models that more accurately reflect the human condition.

\section{Conclusions}

FEA confirmed that vertebral loads can be significantly reduced after augmentation, although the restoration of $\mathrm{VH}$ is different from grades 0 to $3 \mathrm{VH}$ postoperatively. VA procedures have a significant effect on recovery of the biomechanical properties of the vertebral body with an OVCF. Lastly, there is no need for surgeons to pursue anatomic reduction in the clinical treatment of OVCFs with VA if the treatment goal is to only achieve the relief of symptoms.

\section{Abbreviations}

3D: Three-dimensional; ALL: Anterior longitudinal ligament; CL: Capsular ligament; $C$ : Computed tomography; DICOM: Digital Imaging and Communications in Medicine; FEA: Finite element analysis; ISL: Interspinous ligament; LF: Ligamentum flavum; OVCF: Osteoporotic vertebral compression fracture; PKP: Percutaneous kyphoplasty; PLL: Posterior longitudinal ligament; PVP: Percutaneous vertebroplasty; SSL: Supraspinal ligament; VA: Vertebral augmentation; VAS: Visual analog scale; VH: Vertebral height

\section{Acknowledgements}

We thank service@zhuanyejun.com who provided language editing services.

\section{Funding}

This work was supported by the National Natural Science Foundation of China (81560780).

\section{Availability of data and materials}

The datasets supporting the conclusions of this article are included within the article and its additional files

\section{Authors' contributions}

WTZ and DPQ conducted the literature search and selected patients for the experiments. WTZ, DPQ, ZPW, and ZT performed the experiments and extracted data from the studies. WIZ and XGZ conceived the idea of the study, designed the study, and critically revised the manuscript for important intellectual content. The manuscript was written through contributions of all authors. All authors reviewed the paper and approved the final manuscript.

\section{Ethics approval and consent to participate}

This study was granted an exemption from requiring ethics approval by the ethics committee of the Affiliated Hospital of Gansu University of Chinese Medicine. The authors obtained patient consent before enrolling participants in this study.
Consent for publication

Not applicable.

\section{Competing interests}

The authors declare that they have no competing interests.

\section{Publisher's Note}

Springer Nature remains neutral with regard to jurisdictional claims in published maps and institutional affiliations.

\section{Author details}

${ }^{1}$ Gansu University of Chinese Medicine, No. 35, Dingxi East Rd., Chengguan District, Lanzhou 730000, Gansu Province, People's Republic of China. ${ }^{2}$ Affiliated Hospital of Gansu University of Chinese Medicine, No. 735, Jiayuguan West Rd., Chengguan District, Lanzhou 730000, Gansu Province, People's Republic of China. ${ }^{3} Y u n n a n$ University of Traditional Chinese Medicine, No. 1076, Yuhua Rd., Chenggong District, Kunming 650500, Yunnan Province, People's Republic of China.

Received: 9 October 2017 Accepted: 25 January 2018

Published online: 08 February 2018

\section{References}

1. Klazen CA, Lohle PN, de Vries J, et al. Vertebroplasty versus conservative treatment in acute osteoporotic vertebral compression fractures (Vertos II): an open-label randomised trial. Lancet. 2010;376(9746):1085-92.

2. Lee SK, Lee SH, Yoon SP, et al. Quality of life comparison between vertebroplasty and kyphoplasty in patients with osteoporotic vertebral fractures. Asian Spine J. 2014;8(6):799-803.

3. Drampalos E, Nikolopoulos K, Baltas C, et al. Vertebral fracture assessment: current research status and application in patients with kyphoplasty. World J Orthop. 2015;6(9):680-7.

4. Sun H, Li C. Comparison of unilateral and bilateral percutaneous vertebroplasty for osteoporotic vertebral compression fractures: a systematic review and meta-analysis. J Orthop Surg Res. 2016;11(1):156.

5. Odén A, McCloskey EV, Kanis JA, et al. Burden of high fracture probability worldwide: secular increases 2010-2040. Osteoporos Int. 2015:26(9):2243-8.

6. Stevenson M, Gomersall T, Lloyd Jones M, et al. Percutaneous vertebroplasty and percutaneous balloon kyphoplasty for the treatment of osteoporotic vertebral fractures: a systematic review and cost-effectiveness analysis. Health Technol Assess. 2014;18(17):1-290.

7. Tsoumakidou G, Too CW, Koch G, et al. CIRSE guidelines on percutaneous vertebral augmentation. Cardiovasc Intervent Radiol. 2017:40(3):331-42.

8. Ottardi C, La Barbera L, Pietrogrande L, et al. Vertebroplasty and kyphoplasty for the treatment of thoracic fractures in osteoporotic patients: a finite element comparative analysis. J Appl Biomater Funct Mater. 2016;14(2):e197-204.

9. Lieberman $1 H$, Togawa D, Kayanja MM. Vertebroplasty and kyphoplasty: filler materials. Spine J. 2005:5:S305-16.

10. Peppelman WC, Beutler W, Gordon M, et al. Effect of an outer sleeve on an inflatable balloon tamp in terms of height restoration under simulated physiological load. Clin Spine Surg. 2017;30(3):E211-8.

11. Achatz $G$, Riesner HJ, Friemert B, et al. Biomechanical in vitro comparison of radiofrequency kyphoplasty and balloon kyphoplasty. Eur Spine J. 2017; https://doi.org/10.1007/s00586-017-5035-5.

12. Badilatti SD, Christen P, Ferguson SJ, et al. Computational modeling of longterm effects of prophylactic vertebroplasty on bone daptation. Proc Inst Mech Eng H. 2017;231(5):423-31.

13. Rodriguez AJ, Fink HA, Mirigian L, et al. Pain, quality of life and safety outcomes of kyphoplasty for vertebral compression fractures: report of a task force of the American Society for Bone and Mineral Research. J Bone Miner Res. 2017; https://doi.org/10.1002/jbmr.3170.

14. Marcia S, Saba L, Marras M, et al. Percutaneous stabilization of lumbar spine: a literature review and new options in treating spine pain. Br J Radiol. 2016; 89(1065):20150436.

15. Li L, Ren J, Liu J, et al. Results of vertebral augmentation treatment for patients of painful osteoporotic vertebral compression fractures: a meta-analysis of eight randomized controlled trials. PLoS One. 2015;10(9):e0138126.

16. Zysset $P$, Qin L, Lang T, et al. Clinical use of quantitative computed tomography-based finite element analysis of the hip and spine in the management of osteoporosis in adults: the 2015 ISCD official positions-part II. J Clin Densitom. 2015;18(3):359-92. 
17. Hosseini HS, Clouthier AL, Zysset PK. Experimental validation of finite element analysis of human vertebral collapse under large compressive strains. J Biomech Eng. 2014;136(4) https://doi.org/10.1115/1.4026409.

18. Genant HK, Wu CY, van Kuijk C, et al. Vertebral fracture assessment using a semiquantitative technique. J Bone Miner Res. 1993;8(9):1137-48.

19. Liang $D, Y e L Q$, Jiang $X B$, et al. Biomechanical effects of cement distribution in the fractured area on osteoporotic vertebral compression fractures: a three-dimensional finite element analysis. J Surg Res. 2015;195(1):246-56.

20. Polikeit A, Nolte LP, Ferguson SJ. The effect of cement augmentation on the load transfer in an osteoporotic functional spinal unit: finite-element analysis. Spine (PhilaPa 1976). 2003;28:991.

21. Baroud G, Nemes J, Heini $P$, et al. Load shift of the intervertebral disc after a vertebroplasty: a finite-element study. Eur Spine J. 2003;12:421.

22. Goel VK, Kong W, Han JS, et al. A combined finite element and optimization investigation of lumbar spine mechanics with and without muscles. Spine (Phila Pa 1976). 1993;18:1531

23. Zhang L, Yang G, Wu L, Yu B. The biomechanical effects of osteoporosis vertebral augmentation with cancellous bone granules or bone cement on treated and adjacent non-treated vertebral bodies: a finite element evaluation. Clin Biomech (Bristol, Avon). 2010;25:166-72.

24. Denis $F$. The three-column spine and its significance in the classification of acute thoracolumbar spinal injuries. Spine (Phila Pa 1976). 1983;8:817-31.

25. Chung SK, Kim YE. Biomechanical effect of constraint in lumbar total disc replacement: a study with finite element analysis. Spine (Phila Pa 1976). 2009;34:1281-6

26. Rohlmann A, Zander T, Rao M. Applying a follower load delivers realistic results for simulating standing. J Biomech. 2009;42(10):1520-6.

27. Qiu TX, Teo EC, Zhang QH. Effect of bilateral facetectomy of thoracolumbar spine T11-L1 on spinal stability. Med Biol Eng Comput. 2006;44:363-70.

28. Liao JC, Fan KF, Keorochana G, et al. Transpedicular grafting after shortsegment pedicle instrumentation for thoracolumbar burst fracture: calcium sulfate cement versus autogenous iliac bone graft. Spine (Phila Pa 1976). 2010;35:1482-8.

29. Xu G, Fu X, Du C, et al. Biomechanical effects of vertebroplasty on thoracolumbar burst fracture with transpedicular fixation: a finite element model analysis. Orthop Traumatol Surg Res. 2014;100:379.

30. Svensson HK, Olsson L, Hansson T, et al. The effects of person-centered or other supportive interventions in older women with osteoporotic vertebral compression fractures-a systematic review of the literature. Osteoporos Int. 2017; https://doi.org/10.1007/s00198-017-4099-8.

31. PHemama M, El Fatemi N, Gana R. Percutaneous vertebroplasty in Moroccan patients with vertebral compression fractures. Pan Afr Med J. 2017;26:225.

32. Zhang $\mathrm{H}, \mathrm{Xu} \mathrm{C}$, Zhang $\mathrm{T}$, et al. Does percutaneous vertebroplasty or balloon kyphoplasty for osteoporotic vertebral compression fractures increase the incidence of new vertebral fractures? A meta-analysis. Pain Physician. 2017;20:E13-28.

33. Rotter R, Martin H, Fuerderer $\mathrm{S}$, et al. Vertebral body stenting: a new method for vertebral augmentation versus kyphoplasty. Eur. Spine J. 2010; 19(6):916-23.

34. Guo SM, Luo WJ, Huang YM, et al. Percutaneous vertebroplasty and percutaneous balloon kyphoplasty for osteoporotic vertebral compression fracture: a meta analysis. Indian J Orthop. 2015;49:377-87.

35. Ontario Ministry of Health and Long-Term Care. Percutaneous vertebroplasty for treatment of painful osteoporotic vertebral compression fractures: an evidence-based analysis. Ont Health Technol Assess Ser. 2010;10:1-45.

36. Cho AR, Cho SB, Lee JH. Effect of augmentation material stiffness on adjacent vertebrae after osteoporotic vertebroplasty using finite element analysis with different loading methods. Pain Physician. 2015;18:E1101-10.

37. Wilson DR, Myers ER, Mathis JM, et al. Effect of augmentation on the mechanics of vertebral wedge fractures. Spine (Phila Pa 1976). 2000;25:158-65.

38. Berlemann U, Ferguson SJ, Nolte LP, et al. Adjacent vertebral failure after vertebroplasty. A biomechanical investigation. J Bone Joint Surg Br. 2002;84:748-52.

39. Furtado N, Oakland RJ, Wilcox RK, et al. A biomechanical investigation of vertebroplasty in osteoporotic compression fractures and in prophylactic vertebral reinforcement. Spine (Phila Pa 1976). 2007;32:E480-E87.

40. Kim SH, Kang HS, Choi JA, et al. Risk factors of new compression fractures in adjacent vertebrae after percutaneous vertebroplasty. Acta Radiol. 2004;45:440-5.

41. Hadley C, Awan OA, Zoarski GH. Biomechanics of vertebral bone augmentation. Neuroimaging Clin N Am. 2010;20:159-67.

42. Disch AC, Schmoelz W. Cement augmentation in a thoracolumbar fracture model: reduction and stability after balloon kyphoplasty versus vertebral body stenting. Spine (Phila Pa 1976). 2014;39:E1147-E53.
43. Kim JM, Shin DA, Byun DH, et al. Effect of bone cement volume and stiffness on occurrences of adjacent vertebral fractures after vertebroplasty. J Korean Neurosurg Soc. 2012;52:435-40.

44. Bramanti E, Cervino G, Lauritano F, et al. FEM and von Mises analysis on prosthetic crowns structural elements: evaluation of different applied materials. Sci World J. 2017; https://doi.org/10.1155/2017/1029574.

\section{Submit your next manuscript to BioMed Central and we will help you at every step:}

- We accept pre-submission inquiries

- Our selector tool helps you to find the most relevant journal

- We provide round the clock customer support

- Convenient online submission

- Thorough peer review

- Inclusion in PubMed and all major indexing services

- Maximum visibility for your research

Submit your manuscript at www.biomedcentral.com/submit
) Biomed Central 